lobe. The dilatated left anterior cerebrebral artery and middle cerebral artery are major afferent vessel then drained to the cerebral vein and cortical veins which are enormously dilatated additionally small vessels directly to the vein of Galen and inferior sagittal sinus. Recovery from the first attack of SAH was satisfactory.

Case 5. A girl aged 14, who was found in the bed in comatose state with vomitings and right sided hemiplegia. 5 days after was brought to the hospital and lumbar puncture revealed pinc colored c.s.f. under the pressure of $400 \mathrm{~mm}$. Angiographic study disclosed that a peculiar oval shaped shadow on the supra sellar region located the bifurcation of middle cerebral artery, and several small apots which are probably fractional contast media. The left anterior cerebral artery was shifted to the right. We suspected that this is due to the partially ruptured saccoural aneurysm. The boy recovered satisfactory under the conservative treatment and has been doing fine for these 7 years another attack.

Non traumatic SAH in children is rare and after difficult to evaluate of its origin, that some cases may fall into the category of unknown cause. A complete angiographic evaluation should be carried out since this procedure is not so risky even in young child but technically may be troublesome. To get an accurate angiographic information will make a proper classification whether the case belong to the operable (surgical) SAH or medical SAH.

\title{
h-8. Clinical and Pathological Evaluation upon SAH of Unidentified Etiology
}

\author{
Ryozo Fujinaga, Ikuro Murase, Masaki Moriyama, Daizo Tsukumo, \\ Yoichiro NAKagawa and Takashi Tomita \\ Division of Neurological Surgery, Department of Surgery, Srhool of \\ Medicine, Keio University
}

Out of 342 cases of clinically diagnosed SAH between 1961 and 1968 from Keio University Hospital and other 10 cooperative institutes, forty-five cases $(13.2 \%)$ are screened out in this series in which the cause of bleeding can not be identified by repeated angiography and other examinations.

By detailed analysis of these unidentified cases, the characteristics are lined up as follow in comparison with the cases of verified aneurysm and A-V malformation.

1. Age distribution has two peaks namely in 10-30 and 50-60 years of age whereas the aneurysm in 30-60 and A-V malformation in 10-30 years of age.

2. The episode of $\mathrm{SAH}$ is milder in manifestation and less in the frequency of the recurrence.

3. C.S.F. is more rapidly cleared up.

4. The post-episodic sequella is less and severe neurological deficiencies 
remain rarely even though the initial attack manifests typical SAH in severity.

5. Prognosis is used to be benign and mortality rate is only $2.6 \%$ whereas $25.3 \%$ in aneurysm and $10 \%$ in $\mathrm{A}-\mathrm{V}$ malformation. All cases of $\mathrm{SAH}$ of unidentified etiology in this series survive and return to the previous profession except one.

Other multiple estimated etiologies were proposed by Sahs (1966) however there is no analogous case in our series except for one thrombocytopenic purpura as basic desease. The most possible cause of the unidentified SAH is thought to be the rupture of hidden micro-aneurysm on angiogram exceeding the level of usual angiographical analysis as pointed out by Krayenbuihl or due to the spontaneous thrombus formation in the ruptured aneurysm. As for the latter, we experienced $4 \times 4 \times 3 \mathrm{~mm}$ berry type aneurysm disappeared completely in the course of hospitalization which was clearly demonstrated on repeated angiography.

In addition, we succeeded in verifying the ruptured micropial vessel causing thin hemorrhage in the subarachnoidal space in autopsy terminated by the rupture of the another coexisting berry type aneurysm. This also indicates the possibility of such instance in case of the aged group with hypertensive atherosclerotic vascular changes.

\title{
h-9. Subarachnoid Hemorrhage due to rare causes
}

\author{
Junichi Kawafuchi, Tadashi Aiba, Fumikazu Takeda, Ichiro Handa, \\ Hisao YAGI and Norio Kono \\ Dept. of Neurosurgery, Gumma University
}

\section{Discussion to h-9.}

\section{Subarachnoid Hemorrhage Caused by Pituitary Adenoma}

\author{
Hideo Hiratsuka, Akira Kamisasa and Yutaka Inaba \\ Department of Neurosurgery, School of Medicine, Tokyo Medical and Dental University
}

Two cases of subarachnoid hemorrhage consequent to pituitary adenoma are reported. Case 1. A 32 year old female with history of amenorrhea, occasional headache and left visual disturbance was admitted because of sudden onset of severe headache, nausea, vomiting and amblyopia. On admission she was drowsy or stuporous. Neurological examination revealed right sided anosmia, amblyopia, papilledema, no reaction of pupils to light and left abducens paralysis. The neck was stiff to forward flexion. The CSF pressure was raised $(580 \mathrm{mmH} 20)$ 